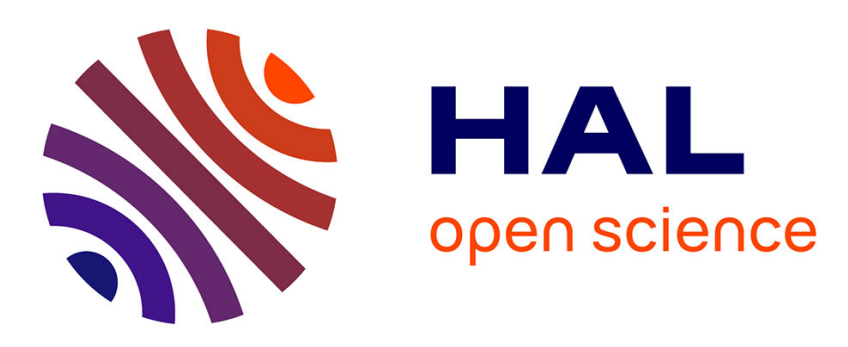

\title{
L'influence d'El Niño et de La Niña sur le niveau de la mer
}

Anny Cazenave, Dieng Habib Boubacar, Simon Munier, Olivier Henry, Benoit Meyssignac, Hindumathi Palanisamy, W. Llovel

\section{- To cite this version:}

Anny Cazenave, Dieng Habib Boubacar, Simon Munier, Olivier Henry, Benoit Meyssignac, et al.. L'influence d'El Niño et de La Niña sur le niveau de la mer. La Météorologie, 2012, 79, pp.34-39. $10.4267 / 2042 / 48511$. hal-01162498

\section{HAL Id: hal-01162498 https://hal.science/hal-01162498}

Submitted on 10 Jun 2015

HAL is a multi-disciplinary open access archive for the deposit and dissemination of scientific research documents, whether they are published or not. The documents may come from teaching and research institutions in France or abroad, or from public or private research centers.
L'archive ouverte pluridisciplinaire $\mathbf{H A L}$, est destinée au dépôt et à la diffusion de documents scientifiques de niveau recherche, publiés ou non, émanant des établissements d'enseignement et de recherche français ou étrangers, des laboratoires publics ou privés. 


\section{L'influence d'El Niño et de La Niña sur le niveau de la mer}

\author{
Anny Cazenave ${ }^{(1)}$, Habib Boubacar Dieng( ${ }^{(1)}$, Simon Munier(1), \\ Olivier Henry ${ }^{(1)}$, Benoit Meyssignac ${ }^{(1)}$, Hindumathi Palanisamy ${ }^{(1)}$ \\ et William Llovel ${ }^{(2)}$ \\ (1) Laboratoire d'études en géophysique et océanographie spatiales (LEGOS) \\ Observatoire Midi-Pyrénées \\ 18 avenue Édouard Belin - 31401 Toulouse Cedex 9, France \\ (2) Jet Propulsion Laboratory, Pasadena, États-Unis
}

\section{Résumé}

Outre une hausse moyenne de l'ordre de $3 \mathrm{~mm}$ par an, le niveau moyen global de la mer présente des fluctuations de quelques millimètres durant les événements El Niño et La Niña. Lors de El Niño, on observe une anomalie positive alors qu'à La Niña correspond une anomalie négative du niveau de la mer. Ces fluctuations du niveau moyen global de la mer sont inversement corrélées aux variations du stock d'eau total sur les continents. Cette observation est en accord avec le fait que, durant El Niño, il pleut davantage sur l'océan que sur les continents, et inversement durant La Niña. Cela suggère que les fluctuations du niveau moyen global de la mer associées aux événements El Niño/La Niña sont plutôt dues à des variations de masse de l'océan qu'à des variations d'origine thermique. Dans cet article, on montre qu'au cours de l'événement El Niño de 1997-1998, l'anomalie positive de masse de l'océan est localisée dans l'océan Pacifique tropical nord. L'excès de masse de cette région compense de manière quasi parfaite le déficit du stock d'eau total des continents à cette période. 'altimétrie spatiale de haute précision a révélé que le niveau moyen global de la mer a monté assez régulièrement depuis début 1993, à la vitesse moyenne de 3,2 $\pm 0,4 \mathrm{~mm}$ par an (Meyssignac et Cazenave, 2012). Cependant, si l'on y regarde de près, on remarque de petites oscillations interannuelles autour de la tendance linéaire (après retrait du cycle saisonnier), dont l'amplitude est de l'ordre de quelques millimètres. Il est assez frappant que le niveau moyen global de la mer présente une anomalie positive assez marquée lors du grand El Niño de 1997-1998. Cela est illustré sur la figure 1 qui présente les fluctuations du niveau moyen global de la mer entre 1993 et 2011

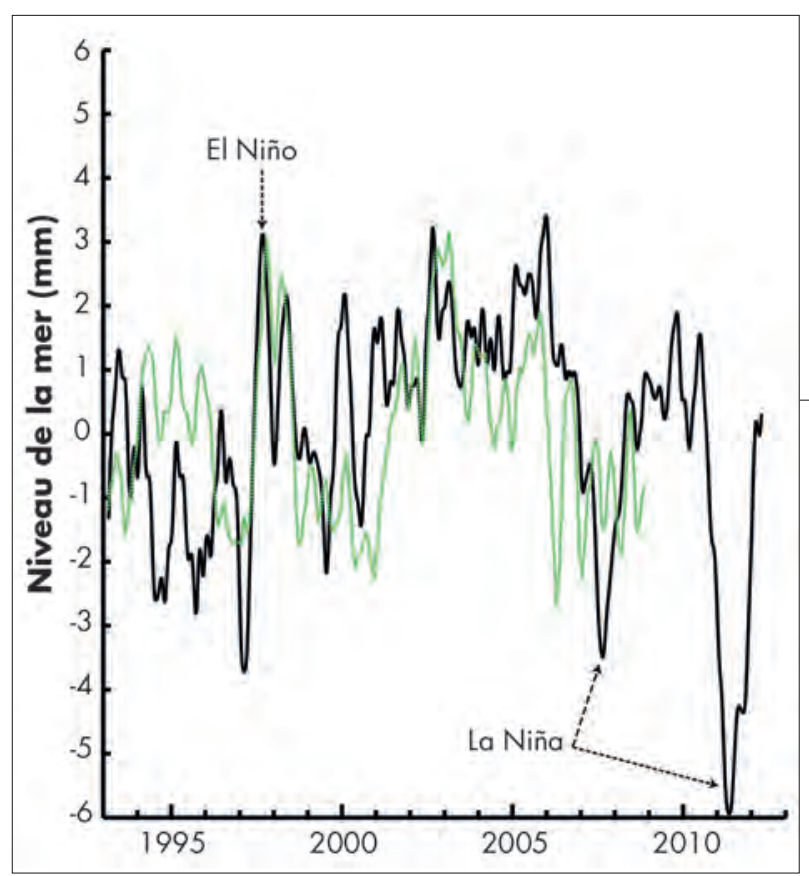

(après retrait d'une tendance linéaire moyenne sur la période). La figure 1 montre aussi des anomalies négatives du niveau moyen global de la mer lors des événements La Niña de 2007-2008 et de 2010-2011. Quelle est la cause de ces anomalies et quel est le lien entre le niveau moyen de la mer et les événements ENSO (El Niño-Southern Oscillation)?

Aux échelles de temps interannuelles à multi-décennales, les principaux phénomènes à l'origine des variations du niveau moyen global de la mer sont :

- l'expansion (ou la contraction) thermique des océans, causée par des variations de la température de la mer (lorsque la température augmente, l'eau de mer se dilate et le niveau de la mer s'élève, et inversement) ;

- l'augmentation (ou la diminution) du contenu en eau des

Figure 1 - En noir : niveau moyen global de la mer mesuré par altimétrie spatiale entre janvier 1993 et décembre 2011 (données du site AVISO www.aviso.oceanobs.com). La tendance linéaire de 3,1 mm/an a été retirée. Les données entre $60^{\circ} \mathrm{S}$ et $60^{\circ} \mathrm{N}$ sont considérées.

En vert : stock d'eau continenta total, estimé à partir du modèle hydrologique ISBA-TRIP de Météo-France, exprimé en équivalent « niveau de la mer ». 


\section{Abstract}

\section{The influence of El Nino and La Nina on sea level}

The detrended global mean sea level displays positive/negative anomalies of a few millimetres amplitude during El Nino/La Nina events that are inversely correlated to total terrestrial water storage variations. This result is in agreement with the observed rainfall deficit/excess over land/oceans during EI Nino (and vice versa during La Nina). It suggests that the positive anomaly observed during El Nino in the global mean sea level is likely due to the ocean mass rather than thermal expansion. A detailed analysis over each oceanic region shows that the global mean sea level anomaly observed during the strong 1997-1998 El Nino resulted from an excess of mass of the north tropical Pacific Ocean with almost perfect compensation with the total terrestrial water deficit during this EI Nino. océans, causée par les apports d'eau douce issus de la fonte des glaces continentales ou associés à des modifications du stock des eaux continentales.

Même si les glaces continentales présentent de petites variations interannuelles, on n'a pas observé de lien net entre leur bilan de masse et ENSO, pour le moment. Il reste donc deux « candidats » pour expliquer les anomalies positives et négatives du niveau moyen global de la mer lors des épisodes ENSO : l'expansion thermique de l'océan et la variation du stock d'eau sur les continents.

\section{Expansion thermique et masse de l'océan durant El Niño}

Au cours des cinq dernières décennies, des mesures de température de la mer ont été collectées par les bateaux, par les bouées océanographiques et, depuis quelques années, par les flotteurs profilants du projet international Argo. Grâce à ces données, les océanographes peuvent estimer la contribution de l'expansion thermique de l'océan au niveau de la mer en intégrant, jusqu'à 700$1000 \mathrm{~m}$ de profondeur, les anomalies de densité de l'eau induites par les variations de température. La figure 2 (courbes du haut) montre l'expansion thermique moyennée sur l'ensemble du domaine océanique, sur la période 1993-2010 (une tendance linéaire moyenne sur la période a été retirée). Le niveau moyen global de la mer (tendance linéaire retirée) y est superposé.
On n'observe pas d'anomalie significative de l'expansion thermique lors de l'épisode El Niño de 1997-1998. On peut donc écarter l'expansion thermique de l'océan comme cause de l'anomalie positive du niveau de la mer observée à cette date.

La figure 2 (courbes du bas) présente les variations du niveau moyen global de la mer (tendance linéaire retirée) et de la masse de l'océan global (calculée par différence entre le niveau moyen global de la mer et l'expansion thermique, après retrait d'une tendance linéaire sur chacune des deux séries temporelles). On note une excellente correspondance entre les deux quantités à l'échelle de temps interannuelle, en particulier lors de l'événement El Niño de 1997-1998. Cela suggère que ce sont plutôt les variations de masse de l'océan, et non celles de l'expansion thermique, qui expliquent les fluctuations observées du niveau moyen global de la mer.

\section{Eaux continentales et niveau de la mer durant El Niño}

Dans une étude récente (Llovel et al., 2011), une équipe du Laboratoire d'études en géophysique et océanographie spatiales (LEGOS) a observé une forte corrélation quantitative entre la variabilité interannuelle du niveau moyen global de la mer (tendance linéaire retirée) et le stock total d'eau dans les bassins fluviaux, en particulier lors de l'événement El Niño de 1997-1998. Il y a donc

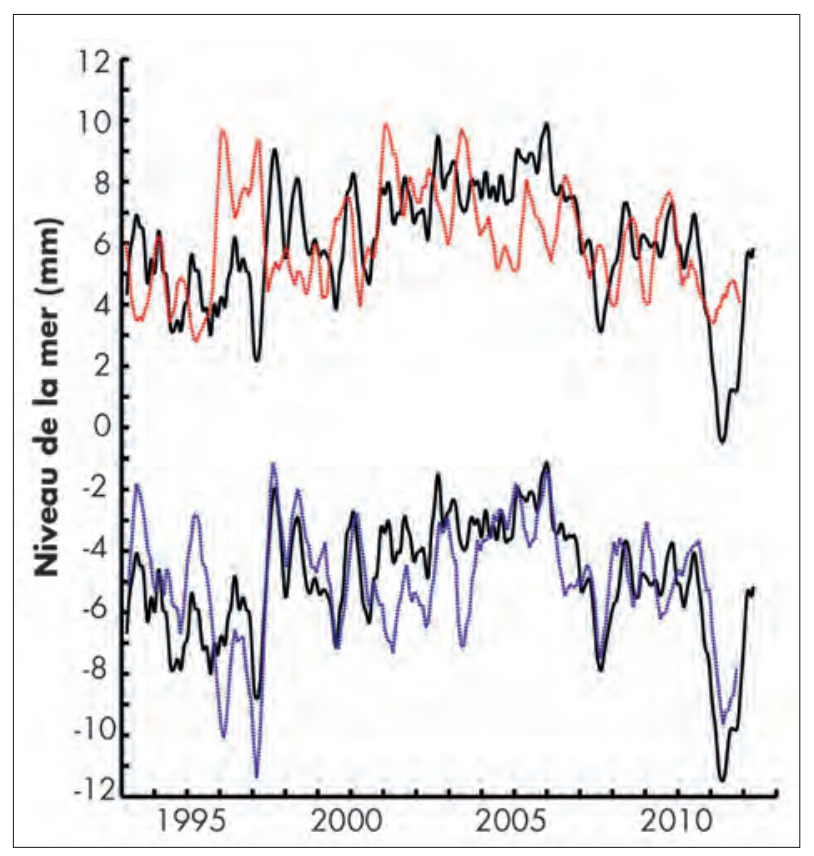

Figure 2 - En haut : en noir, niveau moyen global de la mer mesuré par altimétrie spatiale entre janvier 1993 et décembre 2011, après le retrait de la tendance linéaire (même courbe que sur la figure 1) ; en rouge, expansion thermique moyenne globale, après le retrait de la tendance linéaire (données moyennées entre $60^{\circ} \mathrm{S}$ et $60^{\circ} \mathrm{N}$ d'après la version 6.12 des données de Ishii et Kimoto, 2009).

En bas : en noir, niveau moyen global de la mer (même courbe que ci-dessus et sur la figure 1); en bleu, composante de masse de l'océan estimée par différence entre niveau moyen global de la mer et expansion thermique (les tendances linéaires ont été retirées). 


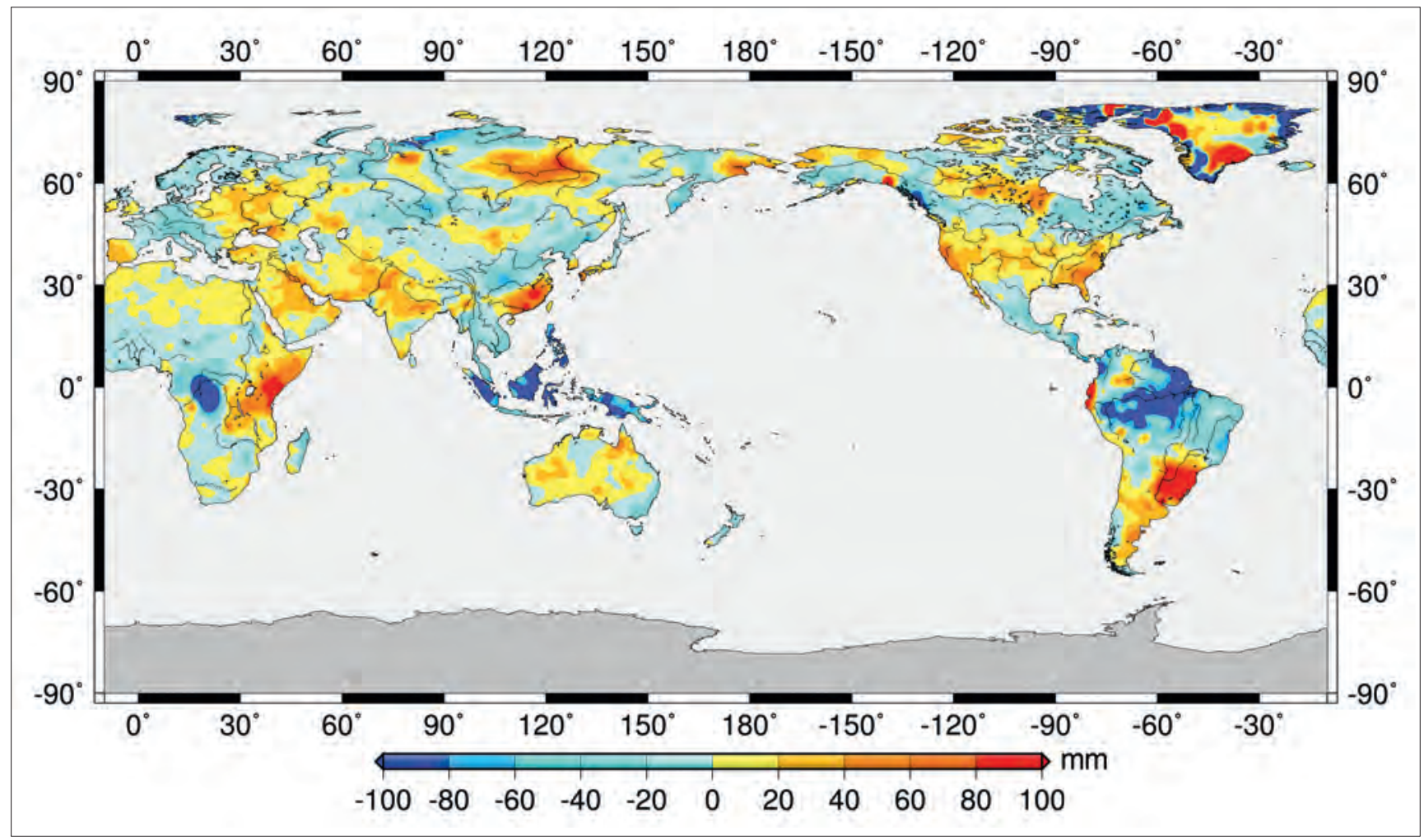

Figure 3 - Carte des anomalies de hauteur d'eau (en mm) sur les terres émergées (d'après le modèle hydrologique ISBA-TRIP de Météo-France). Ces anomalies sont calculées sur une période d’une année (juillet 1997 à juillet 1998) qui coïncide avec l'événement El Niño de 1997-1998.

là une piste pour comprendre le lien entre niveau de la mer et ENSO.

Avec la mission de gravimétrie spatiale GRACE lancée en 2002, il est aujourd'hui possible de mesurer, pour la première fois, les variations spatiotemporelles de la gravité de la terre (Cazenave et Chen, 2010). Aux échelles de temps allant de quelques mois à plusieurs années, ces variations temporelles de gravité résultent principalement des variations de la masse de glace des calottes polaires et des glaciers, ainsi que de la masse d'eau sur les continents en réponse à la variabilité climatique ou aux activités humaines (construction de barrages, irrigation, déforestation, urbanisation, etc.). La résolution spatiale de GRACE (environ $300 \mathrm{~km}$ ) permet de cartographier ces différentes sources et les signaux associés, en particulier les variations des stocks d'eau dans les grands bassins fluviaux (Ramillien et al., 2008). Cependant, pour estimer les variations du stock total d'eau sur les continents avant 2002, il faut faire appel à des modèles hydrologiques. C'est ce qu'ont fait Llovel et al. (2011). Ils ont utilisé les sorties du modèle hydrologique global ISBA-TRIP, développé à Météo-France, dans une version utilisant le forçage météorologique de l'université Princeton entre 1950 et 2008, avec un pas de temps d'un mois et une résolution au sol de $1^{\circ} \times 1^{\circ}$ (voir Alkama et al., 2010). Pour chaque pas de temps, la masse d'eau des différentes couches du sol considérées par le modèle a été moyennée géographiquement sur l'ensemble des terres émergées (à l'exclusion des calottes polaires). Cette masse d'eau a été exprimée en équivalent « niveau de la mer », en pondérant par le rapport des surfaces entre continents et océans, et en multipliant par -1 (pour exprimer le fait qu'un excès d'eau sur les continents correspond à un déficit dans l'océan, et inversement). Sur la figure 1, la série temporelle correspondante est superposée à celle du niveau moyen global de la mer (tendance linéaire retirée). On remarque une correspondance relativement bonne entre les deux quantités lors de l'épisode El Niño de 1997-1998. La corrélation positive entre les deux courbes indique que, pendant cet événement, il y a un excès d'eau dans l'océan et un déficit d'eau sur les continents.

Cela n'est pas vraiment surprenant puisque plusieurs études ont montré que, durant El Niño, il y a davantage de précipitations sur l'océan et moins de pluie sur les continents, en particulier dans les tropiques (Dai et Wigley, 2000 ; $\mathrm{Gu}$ et al, 2007 ; Gu et Adler, 2011). L'étude de Llovel et al. (2011) a par ailleurs montré que la contribution dominante au déficit d'eau continental est celle du bassin de l'Amazone, lors de l'événement El Niño de 1997-1998. Cela est illustré par la figure 3 qui montre les anomalies de stock d'eau dans le sol moyennées sur la période juillet 1997-juillet 1998, d'après le modèle ISBA-TRIP de Météo-France (les données sont exprimées en $\mathrm{mm}$ d'eau, dans un pixel de $1^{\circ} \times 1^{\circ}$ ). Durant cette période qui correspond au El Niño de 19971998 , on voit très bien qu'il y a un fort déficit d'eau dans le bassin de l'Amazone.

\section{Augmentation de la masse de l'océan Pacifique tropical nord durant le El Niño de 1997-1998}

Ce que traduit la figure 1 est essentiellement la conservation de la masse d'eau dans le système Terre à l'échelle de temps interannuelle (en négligeant le réservoir atmosphérique, ce qui est justifié, en première approximation, compte tenu du court temps de résidence de l'eau dans l'atmosphère). Le déficit d'eau dans les bassins fluviaux lors de l'événement El Niño de 19971998 suggère que l'excès d'eau dans l'océan n'est pas causé par le ruissellement des fleuves vers l'océan. Cela est 


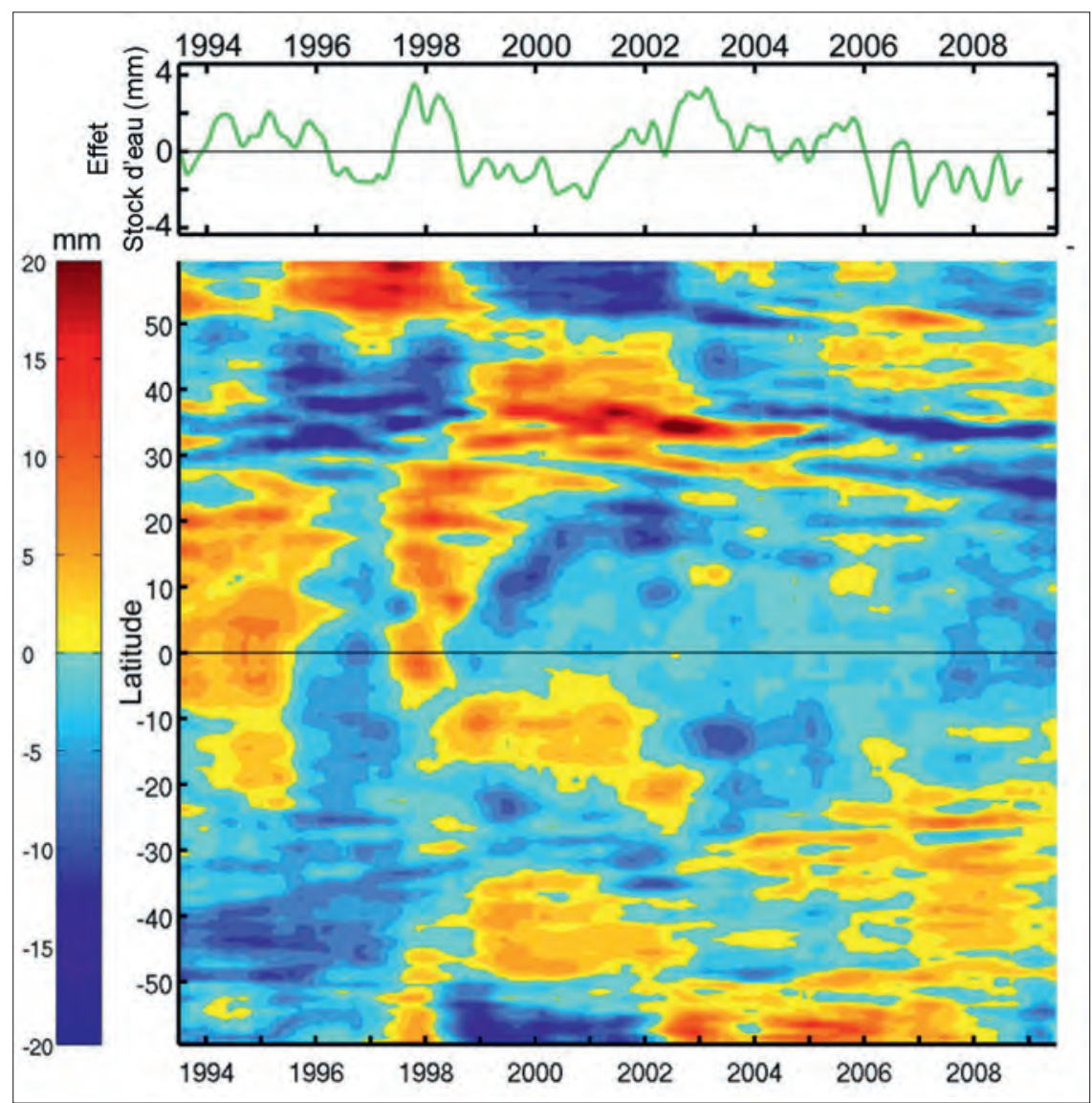

Figure 4 - Diagramme latitude-temps qui représente les variations spatio-temporelles de la masse de l'océan Pacifique moyennée en longitude (entre $120^{\circ} \mathrm{E}$ et les côtes d'Amérique), en fonction de la latitude et du temps. La courbe du haut (en vert) montre l'évolution, en fonction du temps, du stock d'eau continental exprimé en équivalent « niveau de la mer ».

en accord avec les observations qui indiquent un excès de précipitations sur les océans tropicaux. Mais, l'excès de pluie tombée sur l'océan se répartit uniformément sur le domaine océanique en seulement quelques jours. On pourrait donc s'attendre à ce que l'excès de masse de l'océan qui lui est associé soit uniforme géographiquement. C'est que qu'a cherché à vérifier l'équipe du LEGOS dans une autre étude récemment publiée (Cazenave et al., 2012). L'analyse a consisté à estimer, pour chaque océan, la composante « masse de l'océan » par différence entre le niveau moyen de la mer de cet océan, estimé à partir des données d'altimétrie spatiale, et la composante stérique (après retrait des tendances linéaires). La composante stérique représente la somme de l'expansion thermique et des effets de salinité de l'océan. Cette composante a été calculée en utilisant la base japonaise (mise à jour de Ishii et Kimoto, 2009) de données d'anomalies de température et de salinité de l'océan (alors qu'en moyenne globale, la salinité a une influence négligeable sur le niveau de la mer, ce n'est plus vrai à l'échelle régionale et il faut tenir compte des anomalies de salinité). Pour l'océan Atlantique, l'analyse montre que l'essentiel des variations interannuelles du niveau de la mer est d'origine stérique. La masse de l'océan ne présente aucune anomalie remarquable en 1997-1998. La même observation est faite pour l'océan Indien. En revanche, on voit se dessiner une anomalie positive de la masse de l'océan Pacifique lors de l'événement El Niño de 19971998.

Pour cerner plus précisément l'origine géographique de cette anomalie de masse, on a refait la même analyse sur des bandes de latitude de $10^{\circ}$ sur tout l'océan Pacifique. On a aussi réalisé un diagramme qui représente les variations spatio-temporelles de la masse de l'océan Pacifique moyennée en longitude (entre $120^{\circ} \mathrm{E}$ et les côtes d'Amérique) en fonction de la latitude et du temps. Ce diagramme est reproduit sur la figure 4. Il représente également l'évolution, en fonction du temps, du stock d'eau continental exprimé en équivalent « niveau de la mer » (courbe du haut). L'examen de ce diagramme montre bien un excès de masse du Pacifique tropical nord fin 1997-début 1998.

Les deux exercices décrits ci-dessus ont permis de conclure que, durant l'événement El Niño de 1997-1998, l'océan Pacifique présente un excès de masse localisé dans la bande tropicale délimitée par l'équateur et le parallèle $25^{\circ} \mathrm{N}$. La figure 5 (courbes du haut) présente le niveau moyen de la mer dans le

จ Figure 5 - En haut : la courbe noire continue représente le niveau moyen de la mer mesuré par altimétrie spatiale sur le Pacifique tropical nord $\left(0-25^{\circ} \mathrm{N}\right.$ en latitude ; $120^{\circ} \mathrm{E}$ aux côtes américaines, en longitude) ; la courbe noire en tireté représente la hauteur de la mer stérique moyennée sur la même zone.

En bas : en noir, composante de masse du Pacifique tropical nord (même zone que ci-dessus) ; en vert, stock d'eau continental total, estimé à partir du modèle hydrologique ISBA-TRIP, exprimé en équivalent " niveau de la mer ".

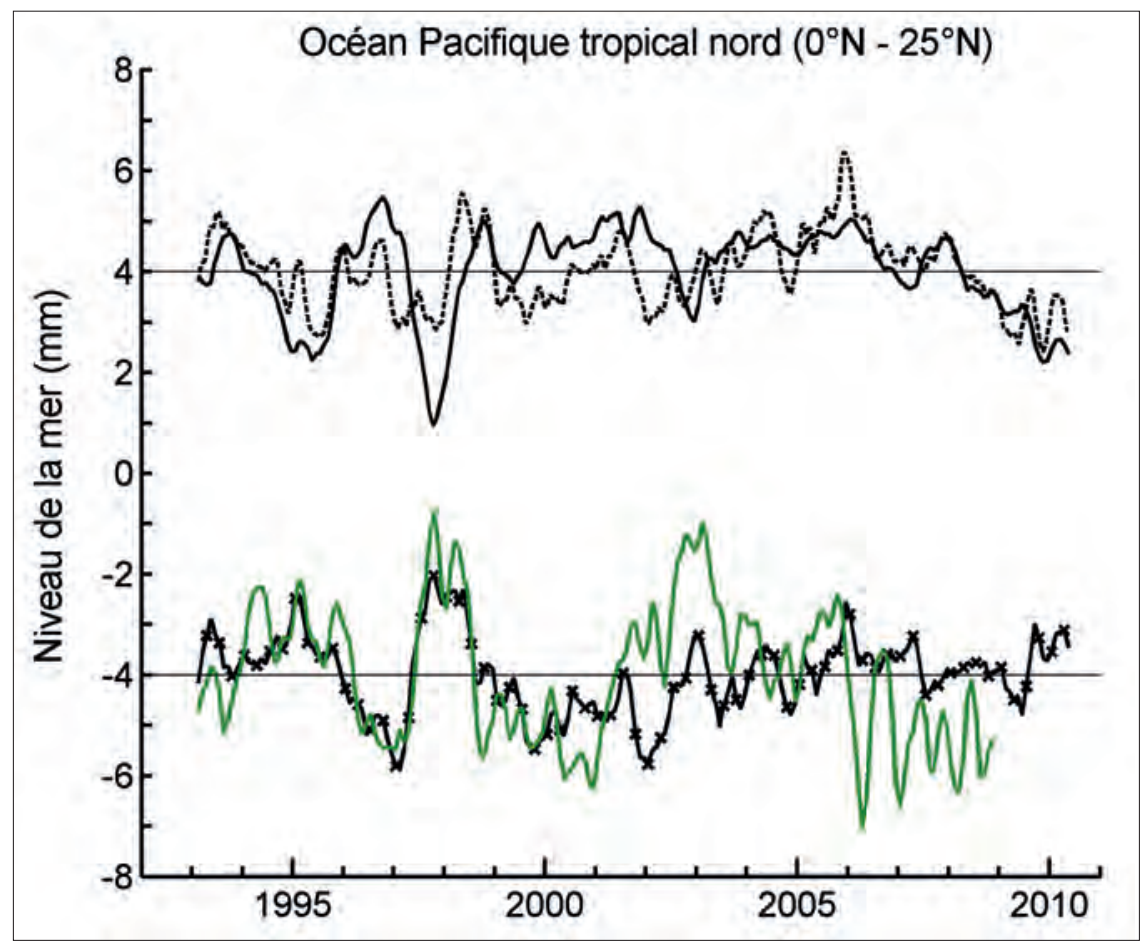


Pacifique tropical nord $\left(0-25^{\circ} \mathrm{N}\right)$, superposé à la composante stérique (tendances retirées pour chaque courbe). Les courbes du bas de la figure 5 correspondent à la composante de masse de l'océan Pacifique tropical nord (estimée par la différence entre les deux précédentes quantités) et à la contribution totale des eaux continentales (exprimée en équivalent " niveau de la mer », comme sur la figure 1). On remarque l'excellente correspondance entre la masse du Pacifique tropical nord et la contribution totale des eaux continentales. Cela traduit une compensation quasi parfaite entre l'excès de masse du Pacifique tropical nord et le déficit d'eau sur les continents lors de l'événement El Niño de 1997-1998. Comment expliquer cette observation ?

\section{Bilan d'eau du Pacifique tropical durant El Niño}

Une estimation du bilan d'eau sur le Pacifique tropical nord pourrait permettre d'y voir un peu plus clair. Le calcul du bilan d'eau sur la région considérée exprime le fait que la dérivée temporelle de la masse d'eau doit être égale à la somme du terme P-E (précipitation $\mathrm{P}$ moins évaporation $\mathrm{E}$ ) et du terme représentant tous les flux d'eau horizontaux. Ce dernier terme comporte lui-même plusieurs composantes : le ruissellement des fleuves (négligé ici car aucun grand fleuve ne se jette dans l'océan dans la zone considérée) et les transports d'eau horizontaux qui entrent et sortent de la zone. Dans un premier temps, on néglige les transports horizontaux. On observe que, si l'on soustrait le terme $(\mathrm{P}-\mathrm{E})$ à la dérivée de la masse d'eau du Pacifique tropical nord, il reste un pic très positif fin 1997-début 1998, comme cela est illustré par la figure 6 (les détails sur les données de précipitation et d'évaporation, utilisées pour ce calcul, se trouvent dans Cazenave et al., 2012). Ce résultat indique que, d'une part, il est nécessaire de faire appel aux transports d'eau horizontaux pour fermer le bilan et que, d'autre part, le flux horizontal net doit être négatif au paroxysme de l'événement El Niño de 1997-1998 (il sort moins d'eau qu'il n'en rentre dans la zone).

Plusieurs études ont montré qu'en période El Niño, le transfert d'eau du Pacifique tropical vers l'océan Indien, via les détroits indonésiens, est réduit

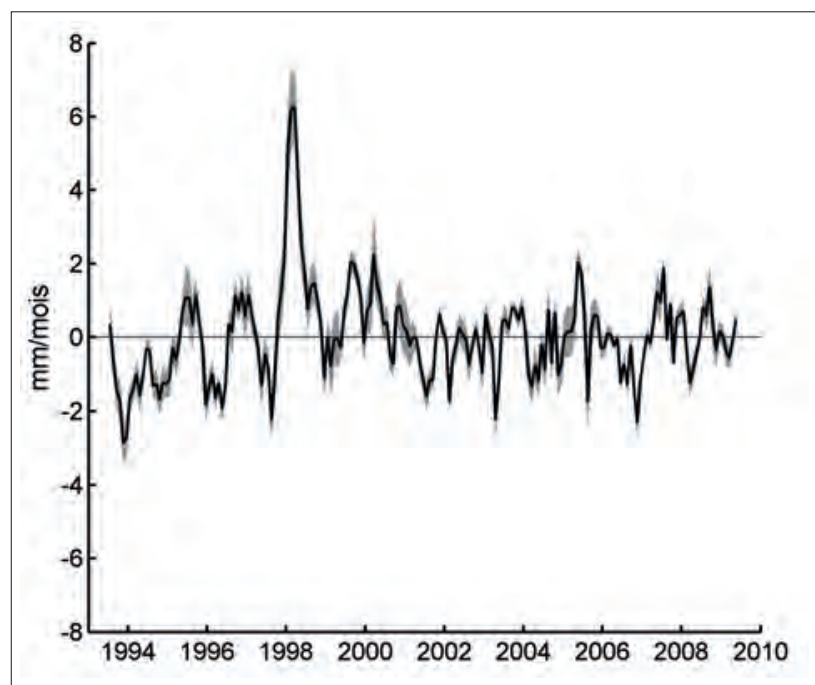

\section{Baisse du niveau de la mer pendant La Niña}

Fin 2007-début 2008, ainsi que fin 2010 début 2011, le niveau moyen global de la mer a baissé temporairement de plusieurs millimètres. Ces anomalies négatives coïncident avec

Figure 6 - Différence entre la dérivée de la composante de masse du Pacifique tropical nord et le terme P-E sur la même zone.

d'un facteur 2. Par exemple, Gordon (2005) a montré que, durant le pic de l'événement El Niño de 1997-1998, le transport d'eau au détroit de Makassar (situé entre Bornéo et Sulawasi) est tombé en dessous de $5 \mathrm{~Sv}$, alors que la moyenne est de 8-12 Sv (voir la figure 7 qui montre les échanges d'eau superficiels entre le Pacifique et l'océan Indien, par les détroits indonésiens). L'écoulement de l'eau du Pacifique tropical nord vers l'océan Indien s'est donc ralenti, conduisant à un excès d'eau temporaire dans le Pacifique. Un bref calcul montre que la réduction du transport d'eau aux détroits indonésiens est du bon ordre de grandeur pour expliquer l'excès de masse du Pacifique tropical nord. Cependant, on ne peut exclure qu'une diminution des transports méridiens y contribue également, notamment au niveau de l'équateur. Une analyse plus approfondie s'impose donc.

Figure 7 - Carte représentant les principaux transports océaniques au niveau des détroits indonésiens. (Source : Gordon, 2005.)

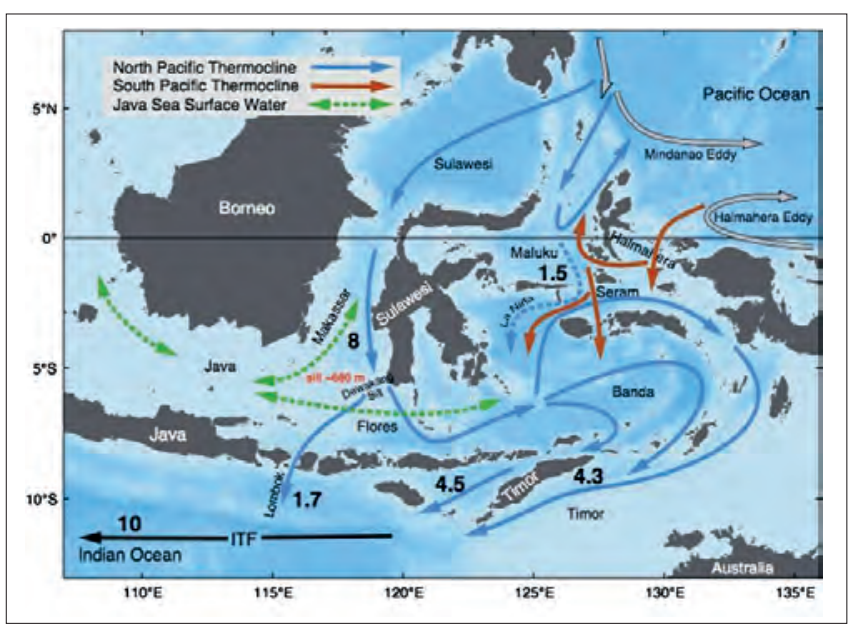

deux épisodes La Niña très intenses (voir figure 1). Durant La Niña de 2010-2011, la baisse du niveau de la mer a atteint $5 \mathrm{~mm}$, ce qui représente une perte d'eau (temporaire) de l'océan de $9000 \mathrm{~km}^{3}$. Comme durant El Niño, le régime des précipitations dans les tropiques pendant La Niña est considérablement modifié. Mais, à l'inverse de ce qui se passe pendant El Niño, il pleut plus sur les continents et moins sur l'océan durant La Niña, ce qui se traduit pas un déficit d'eau dans l'océan. Dans une étude récente basée sur les données de gravimétrie spatiale GRACE, Boeming et al. (2012) ont montré que, lors de l'épisode La Niña de 2010-2011, l'excès d'eau sur les continents est dominé par le bassin de l'Amazone et de l'Orénoque, avec une contribution non négligeable des bassins hydrologiques australiens. Des analyses préliminaires menées au LEGOS semblent indiquer que le déficit d'eau de l'océan se situe principalement dans les océans Pacifique et Indien tropicaux, l'Atlantique ne jouant pratiquement aucun rôle. Mais cela reste à confirmer.

Comme le montre la figure 2 (bas), il faut noter que, durant La Niña de 2010-2011, le déficit de masse de l'océan n'explique que partiellement l'anomalie négative du niveau moyen global de la mer. Il faut donc faire appel à une contribution d'origine thermique lors des phases La Niña. Cela suggère que l'impact de La Niña sur l'océan, sur le niveau de la mer et sur le cycle de 
l'eau n'est pas l'exact symétrique de celui d'El Niño, comme suggéré par Okumura et Deser (2010). Des études devront être menées pour clarifier cette question.

\section{Conclusion}

Jusqu'à présent, les études sur le niveau de la mer ont principalement concerné les causes de la hausse moyenne globale observée depuis quelques décennies, en lien avec le réchauffement climatique. En revanche, les fluctuations interannuelles ont très peu été analysées. Les résultats récents, mentionnés dans cet article, montrent le rôle majeur des événements ENSO sur le niveau moyen global de la mer, via des modifications importantes du cycle hydrologique global. Plus généralement, ces résultats révèlent que les fluctuations interannuelles du niveau de la mer, associées aux événements El Niño, sont essentiellement causées par des changements de la masse des océans. La composante thermique de l'océan joue un rôle mineur.

Les données globales de niveau de la mer par altimétrie spatiale, ainsi que les données de masse de l'océan par gravimétrie spatiale GRACE disponibles depuis 2002, apportent des informations nouvelles et indépendantes des données hydrologiques classiques sur le cycle global de l'eau, en particulier à l'échelle de temps interannuelle.

\section{Bibliographie}

\footnotetext{
Alkama R., B. Decharme, H. Douville, A. Voldoire, S. Tyteca, P. Le Moigne, M. Becker, A. Cazenave et J. Sheffield, 2010 : Global evaluation of the ISBA-TRIP - continental hydrological system. Part 1: Comparison to GRACE Terrestrial Water Storage estimates and in-situ river discharges. J. Hydrometeor., 11, 583-600, doi:10.1175/2010JHM1211.

: Boeming C., J. K. Willis, F. Landerer, S. Nerem et J. Fasullo, 2012 : The 2011 La Nina: so strong, the oceans fell. Geophys. Res. Lett., sous presse.

- Cazenave A., O. Henry, S. Munier, B. Meyssignac, T. Delcroix, W. Llovel et H. Palanisamy, 2012 : ENSO influence on the global mean sea level over $1993-2010$. - Marine Geodesy, doi: 10.1080/01490419.2012.718209, accepté.

- Cazenave A. et J. Chen, 2010 : Time-variable gravity from space and present-day mass redistribution in the Earth system. Earth Planet. Sci. Lett., $298,263-274$.

- Dai A. et T. M. L. Wigley, 2000 : Global patterns of ENSO-induced precipitation. Geophys. Res. Lett., 27, 9, 1283-1286.

: Gordon A. L., 2005 : Oceanography of the Indonesian seas and their throughflow. Oceanography, 18 (4), 14-27.

: Gu G. et R. F. Adler, 2011 : Precipitation and temperature variations on the interannual time scale: assessing the impact of ENSO and volcanic eruptions. J. Climate, 24, 2258-2270.

- Gu G., R. F. Adler, G. J.Huffman et S. Curtis, 2007 : Tropical rainfall variability on interannual to interdecadal and longer time scales derived from the GPCP monthly products. J. Climate, 20, 4033-4046.

- Ishii M. et M. Kimoto, 2009 : Reevaluation of historical ocean heat content variations with varying XBT and MBT depth bias corrections. Journal of Oceanography, 65, : 287-299.

- Llovel W., M. Becker, A. Cazenave, S. Jevrejeva, R. Alkama, B. Decharme, H. Douville, M. Ablain et B. Beckley, 2011 : Terrestrial waters and sea level variations on interannual time scale. Global and Planetary Change, 75, 76-82, doi: 10.1016/j.gloplacha.2010.10.008.

- Meyssignac B. et A. Cazenave, 2012 : Sea level: a review of present-day and recent-past sea level change and variability. J. Geodyn., 58, 96-109.

Okumura Y. et C. Deser, 2010 : Asymmetry in the duration of El Nino and La Nina. J. Climate, 23, 5826-5843.

: Ramillien G., S. Bouhours, A. Lombard, A. Cazenave, F. Flechtner et R. Schmidt, 2008 : Land water contributions from GRACE to sea level rise over 2002-2006. - Global and Planetary Change, 60, 381-392.
} 